\title{
Human DOCK2 Deficiency: Report of a Novel Mutation and Evidence for Neutrophil Dysfunction
}

\author{
Leen Moens ${ }^{1}$ - Mieke Gouwy ${ }^{2}$ - Barbara Bosch ${ }^{3,4}$ • Oleksandr Pastukhov ${ }^{5}$ • Alejandro Nieto-Patlàn ${ }^{6,7,8}$ • Ulrich Siler ${ }^{5}$. \\ Giorgia Bucciol $^{1,3}$ - Djalila Mekahli ${ }^{9,10}$ - François Vermeulen $^{3}$ - Lars Desmet ${ }^{11}$ - Sophie Maebe ${ }^{11}$ - Helena Flipts ${ }^{12}$. \\ Anniek Corveleyn ${ }^{12}$. Despina Moshous ${ }^{13,14}$. Pierre Philippet ${ }^{15}$. Stuart G. Tangye ${ }^{16,17}$ • Bertrand Boisson ${ }^{4,6,7}$. \\ Jean-Laurent Casanova ${ }^{4,6,7,13,18}$ - Benoit Florkin ${ }^{19}$. Sofie Struyf ${ }^{2}$. Janine Reichenbach ${ }^{5,20,21}$. \\ Jacinta Bustamante $\mathrm{e}^{4,6,7,22} \cdot$ Luigi D. Notarangelo ${ }^{23} \cdot$ Isabelle Meyts $^{1,3}$ (D)
}

Received: 7 November 2018 / Accepted: 18 February 2019 / Published online: 5 March 2019

(C) Springer Science+Business Media, LLC, part of Springer Nature 2019

\begin{abstract}
DOCK2 is a guanine-nucleotide-exchange factor for Rac proteins. Activated Rac serves various cellular functions including the reorganization of the actin cytoskeleton in lymphocytes and neutrophils and production of reactive oxygen species in neutrophils. Since 2015, six unrelated patients with combined immunodeficiency and early-onset severe viral infections caused by bi-allelic loss-of-function mutations in DOCK2 have been described. Until now, the function of phagocytes, specifically neutrophils, has not been assessed in human DOCK2 deficiency. Here, we describe a new kindred with four affected siblings harboring a homozygous splice-site mutation (c.2704-2 A > C) in DOCK2. The mutation results in alternative splicing and a complete loss of DOCK2 protein expression. The patients presented with leaky severe combined immunodeficiency or Omenn syndrome. The novel mutation affects EBV-B cell migration and results in NK cell dysfunction similar to previous observations. Moreover, both cytoskeletal rearrangement and reactive oxygen species production are partially impaired in DOCK2-deficient neutrophils.
\end{abstract}

Keywords DOCK2 $\cdot$ neutrophil dysfunction

\section{Introduction}

Dedicator of cytokinesis 2 (DOCK2) is a major guaninenucleotide-exchange factor (GEF) for GTPase-activating proteins, and is primarily expressed in hematopoietic cells [1,2]. It binds and activates Rac through its DOCK homology region domain (DHR) [2]. Activated Rac serves multiple functions downstream of G protein-coupled receptors (GPCR) and antigen and complement receptors, including reorganization of

Mieke Gouwy, Barbara Bosch, Oleksandr Pastukhov, Alejandro NietoPatlàn, Ulrich Siler contributed equally as second authors. Benoit Florkin, Sofie Struyf, Janine Reichenbach, Jacinta Bustamante, Luigi D.

Notarangelo and Isabelle Meyts contributed equally to this work as senior authors.

Electronic supplementary material The online version of this article (https://doi.org/10.1007/s10875-019-00603-w) contains supplementary material, which is available to authorized users.

Isabelle Meyts

isabelle.meyts@uzleuven.be

Extended author information available on the last page of the article the actin cytoskeleton [3-8]. Numerous GEFs can activate Rac and their relative importance depends on the cell type, the ligand, and the downstream effector function in mice and humans [9]. In 2015, Dobbs et al. described five unrelated patients with early-onset invasive viral and bacterial infections caused by bi-allelic loss-of-function (LOF) mutations in DOCK2 [10]. Recently, Alizadeh et al. reported a sixth patient with the same phenotype [11]. The different mutations resulted in absent or markedly reduced levels of DOCK2 protein expression or in expression of a truncated protein lacking the DHR2 domain. Several immunological abnormalities have been documented in DOCK2-deficient patients. These include naïve $T$ cell lymphopenia with reduced $T$ cell receptor excision circles (TRECs) and diminished $\mathrm{T}$ cell proliferation in response to phytohemagglutinin, variable reduction of $\mathrm{B}$ cell numbers, with defective antibody responses despite normal immunoglobulin (Ig) G levels, and impaired NK cell function. Total polymerized filamentous actin (F-actin) was diminished in T-, B-, and NK lymphocytes, and T- and B cell chemotaxis was also impaired by DOCK2 deficiency [10]. Interferon (IFN)-alpha and -lambda production by peripheral blood mononuclear cells (PBMCs) from DOCK2-deficient patients 
was reduced after ex vivo exposure to herpes simplex virus 1 or vesicular stomatitis virus [10]. Moreover, DOCK2deficient simian virus 40 fibroblasts showed increased viral replication and enhanced virus-induced cell death after exposure to encephalomyocarditis virus. Three of the six published patients succumbed to infection (Klebsiella pneumoniae sepsis, viral pneumonitis, and persistent CMV infection); the other three were successfully treated with hematopoietic stem cell transplant (HSCT). Thus, autosomal recessive (AR) DOCK2 deficiency results in a combined immunodeficiency (CID) with additionally impaired innate and cell-intrinsic immunity [10]. Overall, the phenotype of human AR DOCK2 deficiency is reminiscent of observations in Dock $2^{-1-}$ mice [7, 12-14].

Murine Dock $^{-1-}$ neutrophils show also an impaired chemotaxis and reduced reactive oxygen species (ROS) production following $\mathrm{N}$-formylated tripeptide (N-formyl-Met-LeuPhe fMLF) or Phorbol 12-Myristate 13-Acetate (PMA)-induced Rac activation $[15,16]$. The neutrophil chemotaxis and ROS production defects are more pronounced in Dock2/ Dock5 double knockout mice [16]. Pre-treatment of human neutrophils with CPYPP, a small molecule inhibitor of the DHR-2 domain of DOCK proteins and thus of the Rac GEF activity of human DOCK2 and DOCK5, also significantly impaired neutrophil chemotaxis and ROS production [16]. However, to date, no data have been reported on phagocytic cell function in human primary AR DOCK2-deficient neutrophils. Here, we report a new kindred with AR DOCK2 deficiency with four affected children, and demonstrate that this condition is also characterized by neutrophil dysfunction.

\section{Methods}

\section{Study Oversight}

The Ethics Committee of the University Hospitals Leuven approved this study (protocol number: S58466). The study was performed in compliance with the Declaration of Helsinki for research involving human subjects. Written informed consent was obtained from the parents prior to DNA isolation, PBMCs or neutrophil isolation, and Epstein Barr virus (EBV)-transformed B cell production from blood of all family members and healthy controls. For original data, please contact Isabelle.Meyts@uzleuven.be

\section{Whole-Exome Sequencing and Sanger Sequencing}

We performed whole-exome sequencing (WES) on healthy sibling II.1, the index patient (II.6), and his deceased siblings (II.4, II.5) (Fig. 1a). Genomic DNA samples for WES were prepared from heparinized peripheral blood using the QIAamp DNA Blood Midi Kit (QIAGEN, Hilden,
Germany, II.6 or Chemagen DNA extraction kit (PerkimElmer, Boston, MA, USA; II.1, II.4 and II.5). WES libraries were prepared using SureSelect Human V4/V5 enrichment kit (Agilent Technologies, USA). One hundred fifty base pairs paired-end sequencing was performed on the Illumina NovaSeq (Macrogen Europe, Amsterdam, The Netherlands). BWA software was used to align the sequence reads to the Human Reference Genome Build hg19 (http://bio-bwa.sourceforge.net). GATK Unified Genotyper (https://software.broadinstitute.org/gaks.bestpractices) was used to identify single nucleotide variants and insertions/ deletions. ANNOVAR (http://anovar.openbioinformatics.org) was used for annotation. For Sanger sequencing confirmation, genomic DNA samples were prepared from heparinized peripheral blood using the QIAamp DNA Blood Mini kit (QIAGEN, Hilden, Germany). The region of interest around exon 27 of DOCK2 was sequenced using the primers 5 'GGAATTT T T TATGCCCAG-3' and 5'-GCGT GATAGTCCACCCTT-3'. Sanger sequencing was performed on an ABI 3730 XL Genetic Analyzer (Applied Biosystems, Foster City, Calif) at the LGC Genomics Facility in Berlin, Germany. Sequencing data were analyzed using Chromas 2.6.5 (http://www.technelysium.com.au). No DNA sample was available from the deceased sibling (II.2).

\section{Determination of DOCK2 Transcripts by Endpoint PCR and PCR Cloning and Western Blot}

Total RNA was extracted from Ficoll gradient-isolated PBMC using the Absolutely RNA miniprep kit (Agilent Technologies, USA). mRNA was reverse transcribed directly with Superscript Vilo cDNA synthesis kit (ThermoFisher Scientific, Merelbeke, Belgium). PCR was performed using Accuprime I Supermix (ThermoFisher Scientific) and primers (5'-AGTC GAAGTTACTCAGCCAACTCC-3' and 5'-TCTC CATCAAGAAGTCCACAA-3') in the region of interest around exon 25 to 29 of DOCK2. PCR products were analyzed by agarose gel electrophoresis and visualized with GelRed staining (Biotium, Inc., Fremont CA, USA). PCR-purified products (QIAquick PCR purification kit, Qiagen, Hilden, Germany) were cloned using the CloneJET PCR cloning kit (ThermoFisher Scientific). After transformation, pJET1.2 plasmids were isolated with QIAprep Spin miniprep kit (Qiagen, Hilden, Germany) and sequenced at the LGC Genomics Facility in Berlin, Germany. For Western blot, EBV-B cells were lysed with RIPA buffer and immune blotting was performed using anti-DOCK2 (R\&D systems Minneapolis, MN, USA) and anti-GAPDH (Santa Cruz Biotechnology, Heidelberg, Germany) antibodies. Western blot images were captured and quantified with a ChemiDoc MP imager and Image Lab software after 

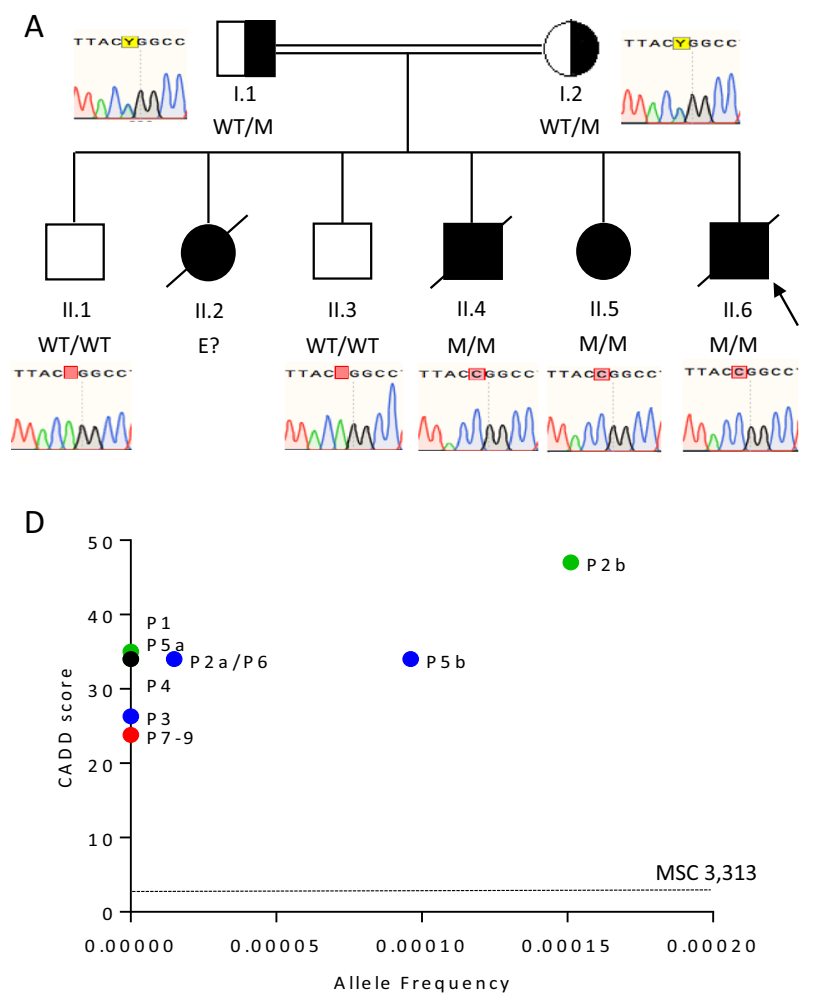

Fig. 1 Novel private bi-allelic mutation in DOCK2 in siblings with SCID and residual thymus. Panel a represents the pedigree of the kindred under study. Circles represent female members and squares male members. A slash through a symbol represents a deceased person. Electropherograms represent Sanger sequencing for evaluation of the segregation of the identified DOCK2 mutation within the family. Patient II.6 is the index patient (indicated with arrow). WT: wild-type, M: mutant, E?: no genetic diagnosis. Panel $\mathbf{b}$ pictures the presence of a normal-sized thymus as visualized by post-natal chest X-ray in II.6. Panel c shows the evolutionary conservation of the nucleotide (red) affected by the identified DOCK2 mutation. Nucleotides in light gray show changes

adding Pierce ECL Western blotting substrate (ThermoFisher Scientific).

\section{EBV-B Cell Migration Assay and NK Cell Degranulation}

Migration of EBV-B cells generated from healthy donors or DOCK2-deficient patient towards different concentrations of CCL20 (500 ng/mL, R\&D Systems) or CXCL12 $(1000 \mathrm{ng} / \mathrm{mL}$, in-house produced and purified as described by Gouwy et al. [17]) was determined using the Multiscreen chemotaxis system as previously described [18]. The Multiscreen plate (Millipore Corporation, Billerica, MA, USA) is a disposable device with a 96well filter plate $(5 \mu \mathrm{m}$ pore size) and a 96 -well receiver plate. Cell migration occurs through the 96-well filter plate in response to a chemotactic gradient. EBV-B cells suspensions $(100 \mu \mathrm{L}$ in 96 -well filter plate at a concentration of $3.5 \times 10^{6}$ cells $\left./ \mathrm{mL}\right)$ and test samples $(150 \mu \mathrm{L}$ in 96-well receiver plate) were diluted in RPMI medium without phenol red and L-glutamine (Lonza, Basel,
B

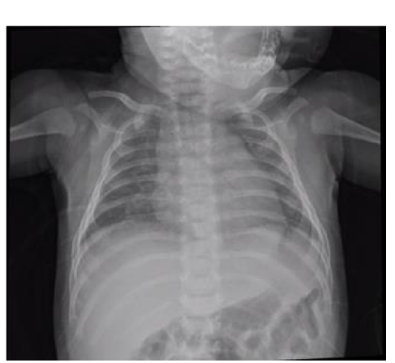

C

$\begin{array}{ll}\text { H. sapiens } & \text { TTACAGGCC } \\ \text { M. musculus } & \text { TTACAGGCC } \\ \text { B. taurus } & \text { TTACAGGCC } \\ \text { S. scrofa } & \text { TTACAGGCC } \\ \text { C. familiris } & \text { TTACAGGCC } \\ \text { G. fortis } & \text { TTGCAGGCT } \\ \text { R. norvegicus } & \text { TTACAGGCC } \\ \text { D. rerio } & \text { TTCCAGGCC }\end{array}$

$E$

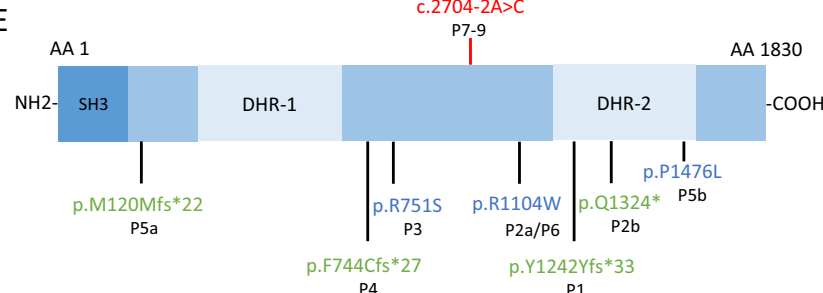

compared to H. sapiens nucleotide sequence (Chr 5: 169267755169267763). Panel d presents a graphic overview of allele frequency and CADD score of published and identified DOCK2 mutations. MSC: mutation significance cutoff. Patients (P1-P5) published in [10] and P6 published in [11] (green: frameshift mutations, blue: missense mutations). P7-9 represent patients described in this study (red). Panel e presents a schematic picture of DOCK2 protein domains and synopsis of diseasecausing DOCK2 mutations [10, 11], green: frameshift mutations, blue: missense mutations; this study, red: splice region mutation. SH3: SRC homology 3 domain, DHR: DOCK homology region domain, AA: amino acid

Switzerland) supplemented with $0.1 \%$ BSA (endotoxin free; Sigma, St Louis, MO, USA). After $4 \mathrm{~h}$ migration at $37^{\circ} \mathrm{C}$, the upper 96-well filter plate was removed and the cells in the lower receiver plate were quantified using the luminescence ATP detection assay system (PerkinElmer Life). The chemotactic activity (CI) was calculated by dividing the luminescence value of the test sample by the luminescence value of the control buffer. For NK cell degranulation analysis, PBMCs were thawed, counted, and seeded $\left(2 \times 10^{6} / \mathrm{mL}\right)$ in IMDM (ThermoFisher Scientific) supplemented with $10 \%$ fetal calf serum. After $3 \mathrm{~h}$ resting $\left(37^{\circ} \mathrm{C}, 5 \% \mathrm{CO}_{2}\right)$, PBMCs were incubated or not (medium condition) 1:1 with human erythroleukemia K562 cells $\left(2 \times 10^{6} / \mathrm{mL}\right)$. Both conditions were supplemented with anti-CD107a-PE (BD Biosciences, Erembodegem, Belgium). Twenty-three hours after stimulation $\left(37{ }^{\circ} \mathrm{C}, 5 \% \mathrm{CO}_{2}\right)$, PBMCs were surface stained with anti-CD3 FITC (BD Biosciences), anti-CD8 APC-H7 (BD Biosciences), and anti-CD56 APC (BD Biosciences). Samples were run on Canto II 
and analyzed with FlowJo cell analysis software (FlowJo, LLC, Ashland, OR, USA.).

Neutrophil Isolation Neutrophils and erythrocytes were separated from PBMCs by density gradient centrifugation $(400 \mathrm{~g}$, $30 \mathrm{~min}, 15^{\circ} \mathrm{C}$, without brake) on Pancoll separating solution (density $1.077 \mathrm{~g} / \mathrm{mL}$; PAN Biotech, Aidenbach, Germany) from EDTA peripheral blood samples. To remove erythrocytes, the neutrophil pellet was mixed with one volume of phosphate-buffered saline (PBS; Lonza, Verviers, Belgium) and one volume of $6 \%$ dextran (Sigma-Aldrich, St. Louis, MO, USA) and erythrocytes were allowed to aggregate and settle during $30 \mathrm{~min}$ at $37^{\circ} \mathrm{C}$. Supernatant was collected and centrifuged $\left(250 \mathrm{~g}, 10 \mathrm{~min}, 15^{\circ} \mathrm{C}\right)$. After washing the pellet with PBS, the remaining erythrocytes in the neutrophil pellet were lysed by hypotonic shock in distilled water for $30 \mathrm{~s}$. After centrifugation and another two washing steps, the neutrophils were suspended in PBS and counted in a hemocytometer (>95\% neutrophils). Alternatively (patient II.5), granulocytes were isolated using the EasySep ${ }^{\mathrm{TM}}$ Direct Human Neutrophil Isolation Kit (StemCell Technologies, Grenoble, France). Both neutrophil isolation steps correlated perfectly in terms of neutrophil analysis afterwards (data not shown) and the choice for either method was based upon the number of samples to handle.

Neutrophil Actin Polymerization Assay Neutrophils $(1 \times$ $10^{6}$ cells $/ \mathrm{mL}, 200 \mu \mathrm{L} /$ well) were adhered on a poly-L-lysine (100 $\mu \mathrm{g} / \mathrm{mL}$, Sigma)-coated 8-well EZ slide chamber (Millipore) for $30 \mathrm{~min}$ at $37^{\circ} \mathrm{C}$. After adherence, the cells were carefully washed with warm RPMI medium (Lonza, Verviers, Belgium) supplemented with 2 mM HEPES (ThermoFisher Scientific) and $0.5 \%$ human serum albumin (Belgium Red Cross) and incubated with medium only or $10^{-8} \mathrm{M}$ fMLF (Sigma-Aldrich, St. Louis, MO, USA) at $37^{\circ} \mathrm{C}$ for $3 \mathrm{~min}$. The reaction mixture was removed and cells were immediately fixed for $15 \mathrm{~min}$ at room temperature (RT) in $2 \%$ paraformaldehyde $(200 \mu \mathrm{L} /$ well $)$. Following fixation, the cells were washed twice with PBS supplemented with $0.01 \%$ Tween-20 (200 $\mu \mathrm{L} /$ well). Next, cells were permeabilized for $5 \mathrm{~min}$ at RT in $0.25 \%$ Triton X-100/PBS, washed again and blocked (3\% BSA + 5\% normal goat serum (SigmaAldrich, St. Louis, MO, USA) in PBS) for $20 \mathrm{~min}$ at RT. Cells were then immediately stained for $30 \mathrm{~min}$ at RT in the dark with rhodamine-phalloidin $(0.17 \mu \mathrm{M}$; ThermoFisher Scientific) and Hoechst ( $5 \mu \mathrm{g} / \mathrm{mL})$. Following staining, cells were washed twice with wash buffer, air-dried, and coverslipped with ProLong Gold Antifade Reagent (ThermoFisher Scientific). Microscopic analysis was performed using an inverted fluorescence microscope (Zeiss Axiovert 200 M, 40× objective; Carl Zeiss, Jena, Germany) using identical acquisition settings for all wells. The fluorescence intensity of polymerized actin was determined in 100 randomly picked cells/condition (Zeiss Axiovision software), averaged, and expressed relative to the medium-treated cells.

Neutrophil Shape Change Assay $3 \times 10^{4}$ neutrophils suspended in Hanks' balanced salt solution (HBSS) containing $10 \mathrm{mM}$ HEPES (without Calcium, Magnesium and Phenol red, shape change buffer, ThermoFisher Scientific) in a 96-well flat bottom plate were supplemented with 1 volume pre-warmed shape change buffer or diluted chemoattractant $\left(10^{-7} \mathrm{M}\right.$ fMLF (Sigma-Aldrich, St. Louis, MO, USA) or $25 \mathrm{ng} / \mathrm{mL}$ CXCL8 (R\&D Systems)). After $5 \mathrm{~min}$ of stimulation, neutrophils were fixed with $100 \mu \mathrm{L}$ of $4 \%$ formaldehyde and two independent observers evaluated each condition in a blinded manner. One hundred cells were counted microscopically (magnification $\times 200$ ) and divided into round cells, cells with protrusions, and elongated cells as described previously [19]. Finally, the average percentage of non-shape changed perfectly round cells, and cells showing membrane protrusions and an irregular cell shape, were calculated [20].

Neutrophil Cytochrome c Reduction Assay $1 \times 10^{6}$ cells were suspended in $1 \mathrm{~mL}$ of HBSS with $\mathrm{Ca}^{2+}$ and $\mathrm{Mg}^{2+}$ (Thermo Fisher Scientific) and incubated in a $10 \mathrm{~mm}$ absorbance cuvette (Hellma, Müllheim Germany) upon continuous stirring at $37^{\circ} \mathrm{C}$ in the presence of $50 \mu \mathrm{M}$ cytochrome $\mathrm{c}$ from equine heart (Sigma). Neutrophil respiratory burst was induced by $100 \mathrm{ng} / \mathrm{mL}$ PMA $1 \mathrm{~min}$ after addition of cytochrome c. The reduction of cytochrome c by extracellularly located superoxide was followed over a time course of at least $10 \mathrm{~min}$ by measuring absorbance at $550 \mathrm{~nm}$ using Nanodrop ${ }^{\mathrm{TM}} \mathrm{One}^{\mathrm{c}}$ (ThermoFisher Scientific).

Intracellular hydrogen peroxide $\left(\mathrm{H}_{2} \mathrm{O}_{2}\right)$ production One milliliter of whole blood was used and erythrocytes were lysed in ammonium buffer. After lysis, cells were washed with PBS $1 \mathrm{X}$ (ThermoFisher Scientific) and resuspended in $1 \mathrm{~mL}$ of PBS, $200 \mu \mathrm{L}$ of the cell suspension was transferred in $5 \mathrm{~mL}$ round-bottom polystyrene tubes (Corning Inc., USA) and kept at $37{ }^{\circ} \mathrm{C}$ in a $5 \% \mathrm{CO}_{2}$ incubator throughout the experiment. ROS production on neutrophils and monocytes was induced by 4-40 and $400 \mathrm{ng} / \mathrm{mL}$ PMA in the presence of $150 \mu \mathrm{L}$ of dihydrorhodamine 123 (DHR 123) at $5 \mathrm{mg} / \mathrm{mL}$ (Sigma). Twenty minutes after PMA treatment, cells were placed on ice and DHR oxidation was measured by flow cytometry using GalliosTM Flow Cytometer (Beckman Coulter, USA) Data were analyzed with Kaluza Flow Cytometry Software (version 1.2; Beckman Coulter).

Superoxide $\left(\mathrm{O}_{2}^{-}\right)$production by EBV-B cell lines $\mathrm{O}_{2}^{-}$production was detected using the Superoxide Anion Assay Kit (Sigma). $3.5 \times 10^{6}$ cells were collected and washed with PBS. Cells were resuspended in $500 \mu \mathrm{L}$ of assay medium and distributed in dark 96-well plates. One hundred 
microliters of working solution containing Luminol reagent with or without PMA at $400 \mathrm{ng} / \mathrm{mL}$ was added following the manufacturer's instructions. Quimio-luminescence was detected during $2 \mathrm{~h}$ using Victor plate reader (Perkin Elmer).

Extracellular $\mathrm{H}_{2} \mathrm{O}_{2}$ Release by EBV-B Cells $\mathrm{H}_{2} \mathrm{O}_{2}$ release was measured using Amplex Red method (ThermoFisher Scientific). Thirty thousand cells were distributed in 96-well plates and were incubated with AmplexRed reagent with or without PMA at $400 \mathrm{ng} / \mathrm{mL}$ following the manufacturer's instructions. Absorbance was measured using Victor plate reader at $590 \mathrm{~nm}$ every $30 \mathrm{~min}$ during $2 \mathrm{~h}$.

\section{Results}

\section{Clinical and Immunological Phenotype of the Affected Children}

The index case was the 6th child (patient II.6) of consanguineous parents of Moroccan descent living in Belgium (Fig. 1a). Patient II.6 was diagnosed at birth with leaky $\mathrm{T}^{-} \mathrm{B}^{+/} \mathrm{NK}^{+}$ SCID according to Primary Immune Deficiency Treatment Consortium (PIDTC) criteria [21] (detailed clinical presentation: Table S1). He suffered from extensive ulcerative perianal dermatitis and presented with E. coli pyelonephritis. Awaiting HSCT, he manifested Omenn syndrome according to PIDTC criteria with $\operatorname{IgE}>40,000 \mathrm{kU} / \mathrm{L}$. He also had nephrotic syndrome. He died from ARDS and capillary leak syndrome as a complication of engraftment syndrome despite treatment with etanercept, tocilizumab, and high-dose steroids. Three of the six siblings had been previously diagnosed with leaky SCID. Patient II.2 succumbed to severe varicella zoster virus infection at 4 months of age. Patient II.4 died from acute respiratory distress syndrome (ARDS) due to alveolar hemorrhage with multi-organ failure at $d+17$ following HSCT. Patient II.5 is alive and well after matched sibling HSCT without conditioning. Interestingly, the affected siblings (II.4, II.5, and II.6, information not available for II.2) had a small to normalsized thymus on post-natal ultrasound and chest X-ray (Fig. 1b). The patients showed profound naïve $\mathrm{T}$ cell lymphopenia and diminished $\mathrm{B}$ cell function and $\mathrm{T}$ cell proliferation (Table S1). A genetic screening panel for known molecular etiologies of SCID had been negative in II.4 in 2014. In particular, no mutations in CORO1A and $C D 3 D$, which result in a PID with similar clinical presentation, were identified [22-25].

\section{Private Bi-allelic Mutation in DOCK2 in Three Siblings with Leaky SCID}

We performed WES on three affected (II.4-6) and one healthy (II.1) sibling from the kindred. DNA from patient II.2 was not available. Under the hypothesis that the disease was inherited as an AR trait with complete penetrance and given the incidence of SCID, we looked for bi-allelic variants with a cutoff allele frequency of $<10^{-4}$ [26]. We identified a homozygous variant in DOCK2 (5:169267759; c.2704-2 A > C) located at an evolutionarily conserved nucleotide at the acceptor splice site of intron 26 as shown by multiple sequence alignment (Fig. 1c) and a combined annotation-dependent depletion (CADD, 23.8) was higher than the mutation significance cutoff (MSC; 3.313 for DOCK2). The effect of the identified variant was predicted to disrupt the acceptor site (position 2) of the start of exon 27 (Human Splice Finder and NNSPLICE 0.9) [27, 28]. The identified variant allele was not found in publicly available databases (dbSNP, ExAC, and gnomAD as assessed in June 2018) or in the Human Genetics of Infectious Diseases Lab in-house database of 4500 WES from patients with severe infections. Furthermore, the variant identified in this kindred was distinct from those detected in the six patients with AR DOCK2 deficiency described to date (Fig. 1d, e, [10,11]). The variant was confirmed by Sanger sequencing and segregated with the clinical and immunological phenotype in the kindred (Fig. 1a). All data suggest that the homozygous variant is deleterious and disease causing.

\section{c.2704-2 A > C in DOCK2 Gives Rise to Alternative Splicing and Causes Complete Loss of Expression of DOCK2}

The observed altered splicing products due to c.2704-2 A $>$ C mutation from patient II.6 were visualized by agarose gel electrophoresis after PCR amplification of exon 26-27 region (Fig. 2a). Skipping of exon 27 (33\%) or retention of 46 nucleotides of intron $26(67 \%)$ was the alternative splicing products of DOCK2 characterized by PCR cloning in PBMCs of patient II.6 (data not shown). These alternative splice products were present together with the wild-type transcript in I.1 (heterozygous carrier). Immunoblot analysis showed that the splicing defects lead to a complete loss of expression of DOCK2 in EBV-B cells derived from patient II.6 (Fig. 2b).

Consistent with this loss of expression, patient II.6 EBV-B cells (II.6) showed a lack of migration to the chemokines CCL20 or CXCL12 compared to EBV-B cells generated from healthy controls (HC, $n=2$ ) (Fig. 2c). Expression of CCR6 and CXCR4, receptors for CCL20 and CXCL12 respectively, on EBV-B cells from patient II.6 was comparable to control EBV-B cells (data not shown, [29]). This indicates that impaired migration does not result from low or absent expression of the chemokine receptors. Furthermore, in line with previous observations [10], we observed aberrant degranulation of DOCK2-deficient NK cells (II.6) upon stimulation with K562 target cells (Fig. 2d, e) compared to NK cells from a 
A

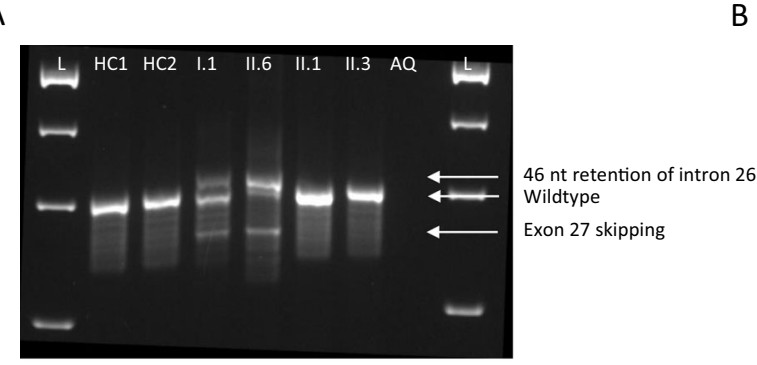

D

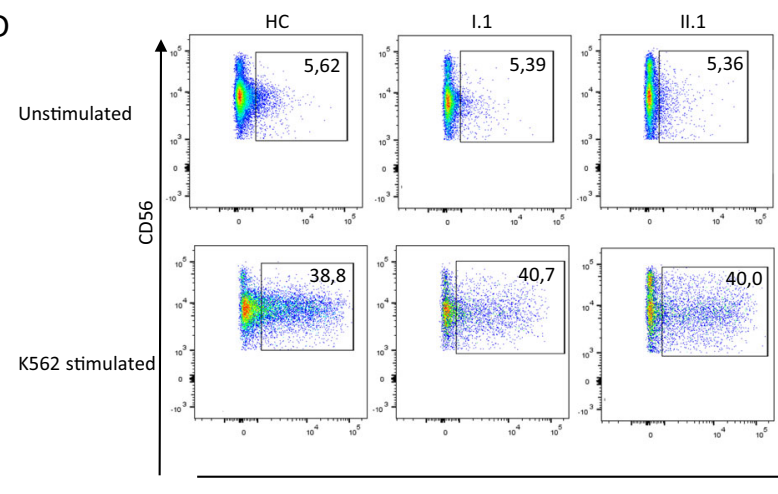

CD107a

Fig. 2 DOCK2 c.2704-2 A $>$ C gives rise to alternative splicing, causes complete loss of expression of DOCK2 and has a functional impact on NK cells and B lymphocytes. Panel a shows the visualization of cDNA fragments (between exon intron boundary 24-25 $(F)$ and 28-29 $(R)$ ) via agarose gel electrophoresis. HC: healthy control, AQ: negative water condition, L: DNA Ladder. Panel b exhibits the DOCK2-specific immunoblot analysis (one out of two representative experiments is shown) of protein lysates from EBV-B cell from two healthy controls (HC) and index patient II.6. Glyceraldehyde-3-phosphate dehydrogenase (GAPDH) was used as protein-loading control. Panel $\mathbf{c}$

healthy control, a healthy wild-type brother (II.1), and a heterozygous DOCK2 mutation carrier (I.1).

\section{DOCK2 Deficiency Impairs F-Actin Polymerization and Shape Change in Neutrophils}

We next studied F-actin polymerization in neutrophils obtained from patient II. 6 at age of 3 months, prior to commencing conditioning for HSCT. DOCK2-deficient neutrophils showed severely impaired F-actin polymerization upon fMLF stimulation (Fig. 3a). F-Actin polymerization in neutrophils of II.5 2 years post-HSCT was similar to that observed in neutrophils from healthy controls, the heterozygous father (I.1) of II.6 as well as a healthy sibling (II.1) not carrying the familial DOCK2 mutation (Fig. 3a). Complementary to these data, neutrophils from patient II.6 showed severely reduced cell protrusions after stimulation with AMLF or CXCL8 (Fig. 3b, c). This profound defect was not present in the transplanted sibling II.5. These data demonstrate that DOCK2-deficient neutrophils have a defect in cytoskeletal rearrangements and shape change, both of which are required for neutrophil polarization and chemotaxis, at least in vitro. shows absent migration in response to CCL20 $(500 \mathrm{ng} / \mathrm{mL})$ or CXCL12 $(1000 \mathrm{ng} / \mathrm{mL})$ in EBV-B cells of patient II.6 compared to EBVtransformed B cell lines of healthy controls (HC, $n=2)$. $P$ value (Mann-Whitney test). Average chemotactic index is shown from three independent experiments performed in triplicate Panel d, e shows impaired NK lymphocyte degranulation, monitored by CD107a surface expression on CD56+ cells, upon stimulation with K562 cells in patient II.6 as compared to healthy control (HC), healthy brother (II.1), and heterozygous DOCK2 mutation carrier (I.1) $(n=1)$. Percentage of $\mathrm{CD} 107 \mathrm{a}^{+} \mathrm{CD} 56^{+}$cells is depicted and noted in the blots

\section{$\mathrm{O}_{2}^{-}$Production Is Impaired in DOCK2-Deficient Neutrophils and EBV-B Cells}

$\mathrm{O}_{2}^{-}$production, as measured by cytochrome $\mathrm{c}$ reduction upon PMA stimulation, was significantly reduced in DOCK2deficient neutrophils from patient II.6 compared to neutrophils from a healthy control (Fig. 4a). In contrast, $\mathrm{H}_{2} \mathrm{O}_{2}$ production as measured by the DHR assay was similar for neutrophils for the healthy control, patient II.6, and a healthy sibling not carrying the familial DOCK2 mutation (II.1) (Fig. 4b). The same observation was made for monocytes (Fig. S1). We also tested the respiratory burst in EBV-B cells, which express NADPH oxidase complex albeit at a lower level than neutrophils [30]. $\mathrm{O}_{2}^{-}$production as measured by the Superoxide Anion assay, after PMA stimulation in EBV$\mathrm{B}$ cells, was severely reduced in patient II.6 compared to the healthy control and resembled lack of $\mathrm{O}_{2}{ }^{-}$production by EBV-B cells from a patient with X-linked chronic granulomatous disease (Fig. 4c). Moreover, in patient II.6's EBV-B cells, the extracellular $\mathrm{H}_{2} \mathrm{O}_{2}$ production upon PMA stimulation was delayed and reduced compared to healthy control as measured by Amplex Red (Fig. 4d). 

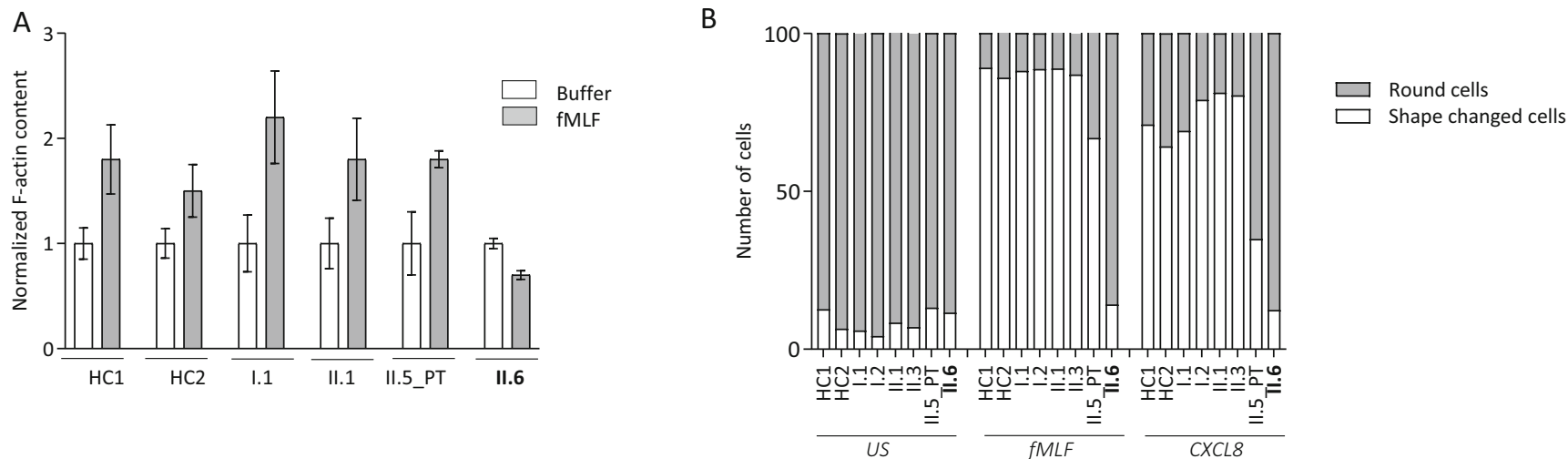

C
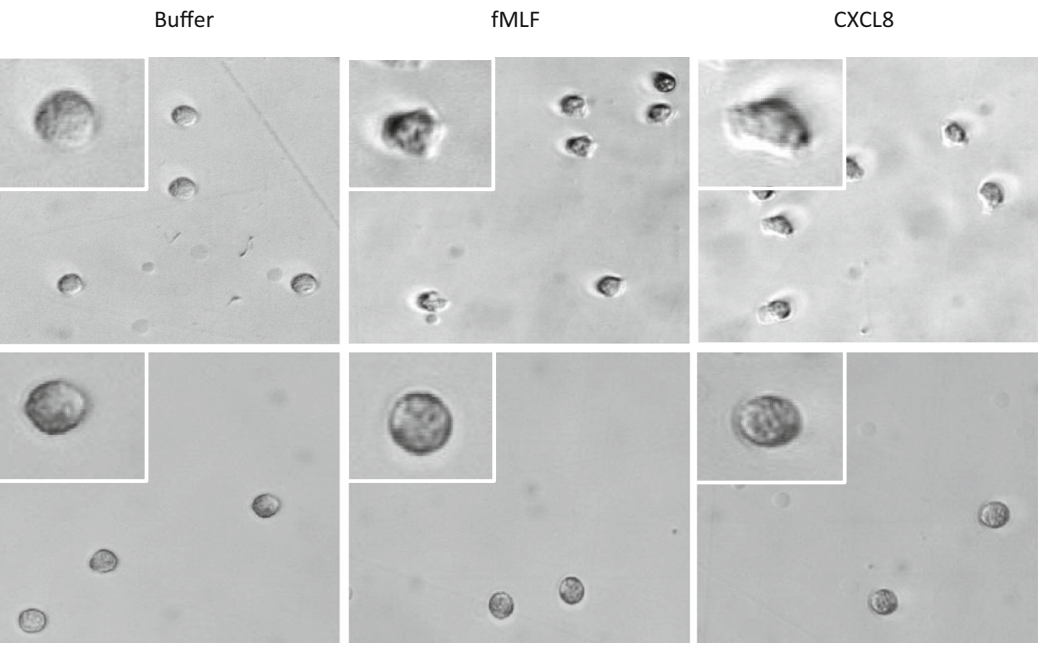

Fig. 3 Impaired actin polymerization and cell shape formation in neutrophils from DOCK2-deficient patient II.6. Panel a presents the normalized filamentous actin polymerization (F-actin) in unstimulated neutrophils (white bar) or $10^{-8} \mathrm{M}$ fMLF (gray bar) for $3 \mathrm{~min}$ followed by fixation. Microscopic pictures were analyzed for mean fluorescent intensity (MFI) of intracellular F-actin. Panel b shows the neutrophil

shape changes after stimulation (fMLF $\left(10^{-7} \mathrm{M}\right)$ or CXCL8 (25 ng/ $\mathrm{mL})$ ) compared to unstimulated cells. After $5 \mathrm{~min}$, neutrophils were fixed and changes in cell shape were evaluated microscopically. Two hundred cells/condition were counted. Microscopic pictures from $\mathrm{HC} 1$ and II.6 are presented in panel c. PT: post-HSCT, US: unstimulated

\section{Discussion}

We report a kindred with AR DOCK2 deficiency caused by a novel homozygous splice-site mutation leading to complete loss of expression of DOCK2 protein. Early-onset severe viral infections, T cell lymphopenia, and functional defects of T-, B- and NK cells are the predominant immunophenotype of DOCK2 deficiency (Table S1) [10, 11]. TRECs were absent in two of the patients reported by Dobbs et al. [10] and in index patient II.6. The DOCK2-deficient patients reported here all fulfill the diagnostic criteria of leaky SCID or Omenn syndrome. Thymic shadow was present on chest X-ray, whereas $\mathrm{T}$ cell lymphopenia in $\mathrm{T}^{-} \mathrm{B}^{+/} \mathrm{NK}^{+} \mathrm{SCID}$ conditions is usually associated with a small or undetectable thymus [31]. Two other (S)CID conditions with detectable thymus, Coronin$1 \mathrm{~A}$ deficiency $[22,23,25]$, and $\mathrm{CD} 3 \delta$ deficiency [24] have been reported. Deficient emigration of mature thymocytes in response to CCL19 has been reported in organ cultures of Dock $2^{-/}$-deficient mice [7]. We hypothesize that the naïve

T cell lymphopenia in the presence of a thymus in DOCK2deficient patients results at least in part from the impaired release of mature $T$ cells from the thymus due to dysregulation of the actin cytoskeleton. DOCK2 deficiency should therefore be considered in the differential diagnosis of SCID and of Omenn syndrome.

Among the five patients included in the first report of human DOCK2 deficiency, one died of Klebsiella pneumoniae sepsis at 20 months of age [10]. The authors suggested that DOCK2 deficiency may also affect phagocytic cell function. Dock $2^{-1-}$ mice are uniquely susceptible to Citrobacter rodentium infection, with impaired recruitment of neutrophils and macrophages to the infected organs, leading to death in a significant proportion of mice [32]. In one of the patients reported here, the extensive perianal dermatitis may allude to a potential phagocyte dysfunction. The nephrotic syndrome and the massive pulmonary inflammation with hemorrhage and ARDS upon engraftment were also particular, though a clear link with neutrophil dysfunction prior to HSCT is 
A

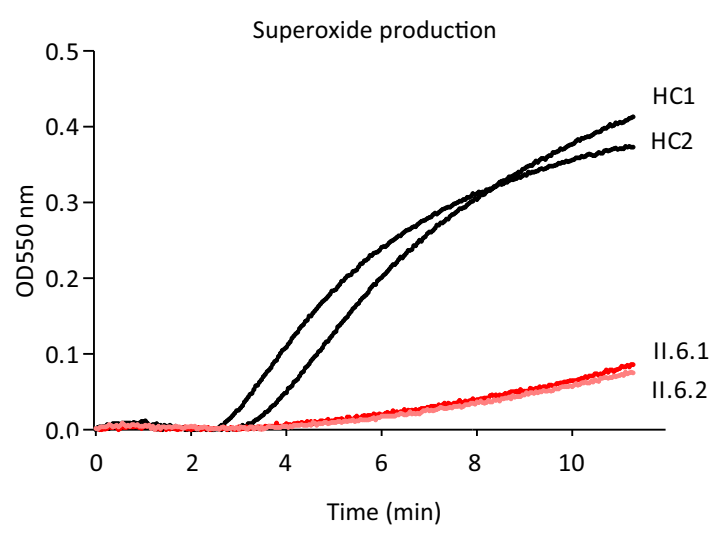

C

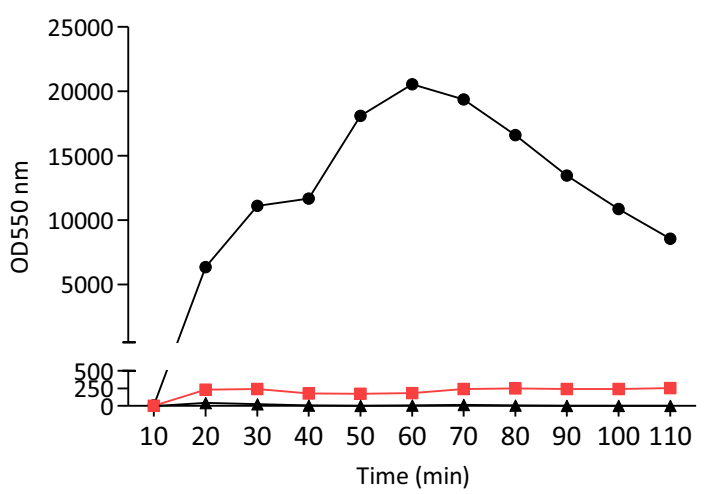

Fig. 4 The respiratory burst is impaired in DOCK2-deficient neutrophils and EBV-B cells. Panel a presents the quantification of superoxide production by neutrophils after stimulation with PMA in cytochrome $\mathrm{c}$ reduction assay. Analysis was run in duplicate for a healthy control (HC, black) and for II.6 (red), indicated as traces HC1, HC2, and II.6.1 and II.6.2 accordingly. Panel b shows the DHR oxidation after PMA stimulation in neutrophils from healthy control (HC), patient II.6, and healthy sibling II.1 measured via flow cytometry. White peaks are unstimulated cells and gray peaks are stimulated cells. Panel $\mathbf{c}$ pictures

unclear. Nevertheless, the present study shows that human DOCK2 deficiency leads to impaired F-actin polymerization and shape change in neutrophils in response to chemoattractants. These processes are essential for neutrophil chemotaxis and migration. Due to sampling limitations, we were unable to perform in vitro chemotaxis assays with neutrophils, but it is likely that these would also be impaired. Indeed, similar results have been reported in $D o c k 2^{-} \Gamma$ mice and in human neutrophils treated with the DOCK $2 / 5$ inhibitor CPYPP [16].

Next, we show an impaired respiratory burst activity in neutrophils from patient II.6, as measured by cytochrome c reduction after PMA stimulation. Reduced $\mathrm{O}_{2}^{-}$production was also found in the patient's EBV-B cells. On the other hand, the DHR assay in DOCK2-deficient neutrophils and
B
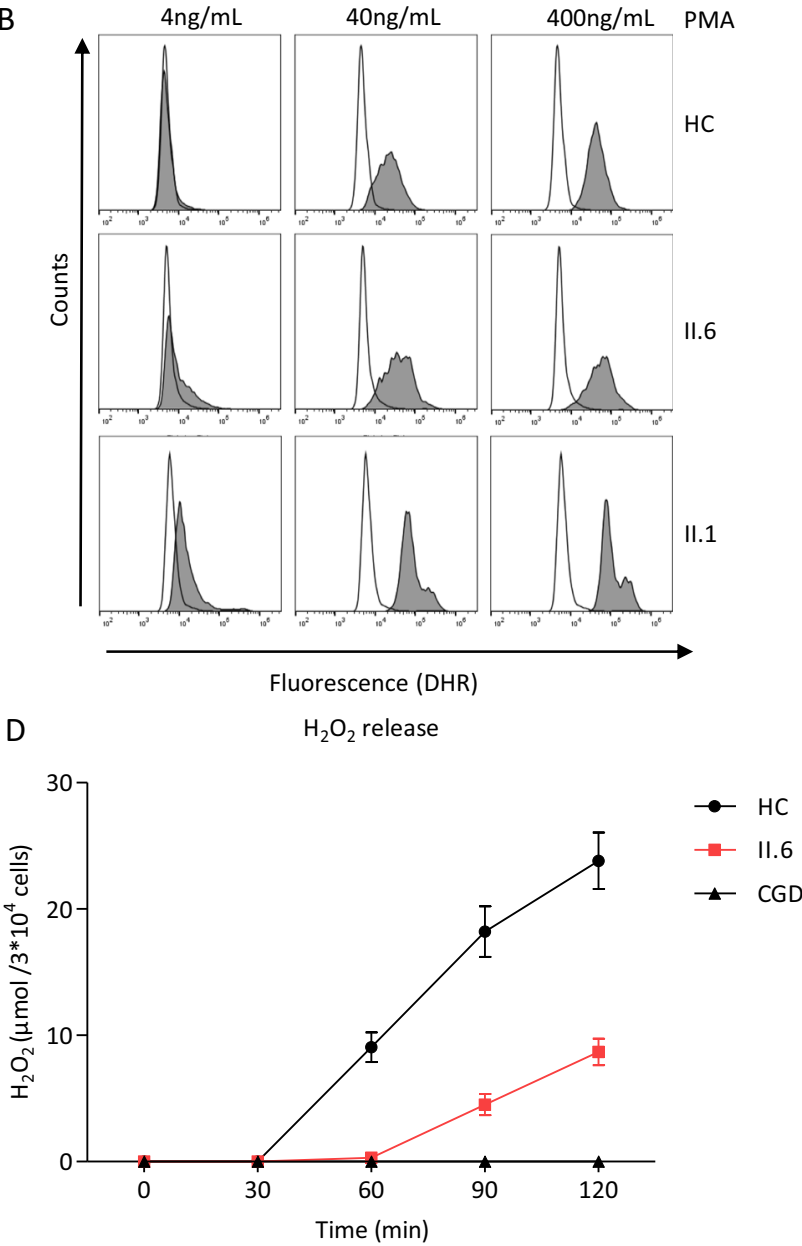

the superoxide production measured by Luminol assay in EBV-B cells after PMA stimulation from healthy control (HC), patient II.6, and from a patient with chronic granulomatous disease (CGD, negative control). One representative experiment out of two is shown. Panel d presents the $\mathrm{H}_{2} \mathrm{O}_{2}$ release after PMA stimulation in EBV-B cells from a healthy control (HC), patient II.6, and from a patient with chronic granulomatous disease (CGD, negative control) using the Amplex Red assay. One representative experiment out of two is pictured

monocytes from patient II.6 was normal in response to PMA (Fig. 4b, Fig. S1). It is important to realize that the DHR assay is a screening assay of respiratory burst activity and not an absolute measurement of hydrogen peroxide production, even more so when using only PMA as a stimulus [33]. The patient was off steroids at the time of sampling. Moreover, the decreased cytochrome c reduction in EBV-B cells suggests that the defect in respiratory burst activity in the patient's neutrophils is a not a secondary event. The description of NADPH oxidase in EBV-B cells and monocytes next to neutrophils is relevant as there may be differential expressivity of a phenotype depending on the cell type studied.

Rac-GTP is part of the assembled NADPH oxidase complex, which acts as a molecular switch to activate 
one of the oxidase subunits, p67phox (NCF2) [34, 35]. Thus, impaired activation of Rac in absence of DOCK2 leads to reduced NADPH oxidase activity by affecting the catalytic activity of the enzyme. We can also presume that due to incomplete activation of Rac and perhaps other small GTPases, the cytoskeleton rearrangement may differ in DOCK2-deficient neutrophils and EBV-B cells as compared to control cells, leading to impaired accumulation of active NADPH oxidase complexes at the cell surfaces and giving rise to the observed impaired respiratory burst at least in vitro. How/if the cytoskeleton regulates enzyme assembly is less well understood. Another possible explanation is the site of $\mathrm{O}_{2}{ }^{-}$detection. DHR is oxidized intracellularly, whereas all other assays used for this project detect oxidase activity extracellularly [30]. Altogether, these data support a role for DOCK2 and the DOCK2$\mathrm{Rac}$ axis and the actin cytoskeleton in the $\mathrm{O}_{2}{ }^{-}$forming respiratory burst activity following stimulation of GPCRs. Unfortunately, we were not able to study the respiratory burst activity in response to other stimuli due to the patient's critical clinical situation and start of conditioning regimen. Indeed, NADPH oxidase activity in response to complement opsonized bacteria may well be normal as these stimuli are expected to activate VAV family and other GEFs via non-G protein-coupled receptors $[4,9]$. The clinical phenotype in DOCK2 deficiency is far less suggestive of neutrophil dysfunction than the phenotype of patients with dominant negative mutations in $R A C 2$, who present with omphalitis and soft tissue abscesses, probably due to the dominant negative RAC2 protein affecting also the GTPase activity of the ubiquitously expressed RAC1 [34-37]. Interestingly, the neutrophil dysfunction phenotype was also modest in two patients with homozygous loss-of-function mutations in $R A C 2$ [38]. Nevertheless, it is prudent to take the at least partially impaired neutrophil chemotaxis and respiratory burst activity into account in DOCK2-deficient patients. Finally, the finding of neutrophil dysfunction in human DOCK2 deficiency confirms the impact of deleterious mutations in genes related to the actin cytoskeleton on the normal function of human myeloid and lymphoid immune cells $[8,34-37,39-46]$.

Acknowledgments We are indebted to the parents and patients, who gave us their ultimate trust. We are indebted to the entire department of pediatric hemato-oncology for their contribution to clinical care. This research was in part supported by Fonds Wetenschappelijk Onderzoek Vlaanderen Project G0C8517N and by the VIB Grand Challenges Program. LDN is supported by the Division of Intramural Research, National Institute of Allergy and Infectious Diseases, National Institutes of Health. ANP was supported by the CONACYT National PhD Fellowship Program. The HGMI laboratory was funded in part by the National Institute of Allergy and Infectious Diseases, The Rockefeller University, St. Giles Foundation, Institut National de la Santé et de la Recherche Médicale (INSERM), Paris Descartes University, the
Integrative Biology of Emerging Infectious Diseases Laboratory of Excellence (ANR-10-LABX-62-IBEID), and the French National Research Agency (ANR) under the "Investments for the future" program (ANR-10IAHU-01).

OP is funded by Forschungskredit of Zürich University (Grant number FK-17-053). JR is funded by Swiss National Science Foundation (grant no: 31003A_182424) Hochspezialisierte Medizin Schwerpunkt Immunologie (HSM-2-Immunologie) Uniscientia Foundation Clinical Research Priority Program ImmuGene of the University of Zurich.

Authorship Contributions LM, IM, LDN, JB, JLC, JR, MG, SS, US, ANP, OP, BB, BeBO, GB contributed to study design and/or performed experiments. LM, IM, JB, LDN, JLC, BB, ANP, SGT, GB, DeMo, JR, $\mathrm{MG}$, contributed to writing the paper.

FV, BF, PP, SM, LD, DM, IM, AC, contributed to data and material collection and clinical care.

All authors have read and approved the final version of the manuscript.

\section{Compliance with Ethical Standards}

Conflict of Interest IM is holder of an institutional CSL-Behring Chair in Primary Immunodeficiencies and is supported by the Jeffrey-Modell Foundation. The other authors declare that they have no conflict of interest.

Informed Consent Informed consent was obtained from all individual participants included in the study.

Publisher's Note Springer Nature remains neutral with regard to jurisdictional claims in published maps and institutional affiliations.

\section{References}

1. Nishihara H, Kobayashi S, Hashimoto Y, Ohba F, Mochizuki N, Kurata T, et al. Non-adherent cell-specific expression of DOCK2, a member of the human CDM-family proteins. Biochim Biophys Acta. 1999;1452(2):179-87.

2. Gadea G, Blangy A. Dock-family exchange factors in cell migration and disease. Eur J Cell Biol. 2014;93(10-12):466-77.

3. Bokoch GM. Regulation of innate immunity by Rho GTPases. Trends Cell Biol. 2005;15(3):163-71.

4. Heasman S, Ridley AJ. Mammalian Rho GTPases: new insights into their functions from in vivo studies. Nat Rev Mol Cell Biol. 2008;9(9):690-701.

5. Wang J, Xu L, Shaheen S, et al. Growth of B cell receptor microclusters is regulated by PIP2 and PIP3 equilibrium and Dock2 recruitment and activation. Cell Rep. 2017;21(9):2541-57.

6. Malhotra S, Kovats S, Zhang W, Coggeshall KM. B cell antigen receptor endocytosis and antigen presentation to T cells require Vav and dynamin. Biol Chem J. 2009;284(36):24088-97.

7. Fukui Y, Hashimoto O, Sanui T, Oono T, Koga H, Abe M, et al. Haematopoietic cell-specific CDM family protein DOCK2 is essential for lymphocyte migration. Nature. 2001;412:826-31.

8. Pfajfer L, Mair NK, Jiménez-Heredia R, et al. Mutations affecting the actin regulator WD repeat-containing protein 1 lead to aberrant lymphoid immunity. J Allergy Clin Immunol. 2018;S00916749(18):30694.

9. Pantarelli C, Welch HCE. Rac-GTPases and Rac-GEFs in neutrophil adhesion, migration and recruitment. Eur J Clin Investig. 2018;48:e12939. 
10. Dobbs K, Domínguez Conde C, Zhang SY, Parolini S, Audry M, Chou J, et al. Inherited DOCK2 deficiency in patients with earlyonset invasive infections. N Engl J Med. 2015;372(25):2409-22.

11. Alizadeh Z, Mazinani M, Shakerian L, Nabavi M, Fazlollahi MR. DOCK2 deficiency in a patient with hyper IgM phenotype. J Clin Immunol. 2018;38(1):10-2.

12. Sanui T, Inayoshi A, Noda M, et al. DOCK2 is essential for antigeninduced translocation of TCR and lipid rafts, but not PKC-theta and LFA-1, in T cells. Immunity. 2003;19(1):119-29.

13. Nombela-Arrieta C, Lacalle RA, Montoya MC, et al. Differential requirements for DOCK2 and phosphoinositide-3-kinase gamma during T and B lymphocyte homing. Immunity. 2004;21(3):429-41.

14. Sakai Y, Tanaka Y, Yanagihara T, Watanabe M, Duan X, Terasawa $\mathrm{M}$, et al. The Rac activator DOCK2 regulates natural killer cellmediated cytotoxicity in mice through the lytic synapse formation. Blood. 2013;122(3):386-93.

15. Kunisaki Y, Nishikimi A, Tanaka Y, et al. DOCK2 is a Rac activator that regulates motility and polarity during neutrophil chemotaxis. J Cell Biol. 2006;174(5):647-52.

16. Watanabe $\mathrm{M}$, Terasawa M, Miyano K, et al. DOCK2 and DOCK5 act additively in neutrophils to regulate chemotaxis, superoxide production, and extracellular trap formation. J Immunol. 2014;193(11):5660-7.

17. Gouwy M, Struyf S, Mahieu F, et al. The unique property of the CC chemokine regakine-1 to synergize with other plasma-derived inflammatory mediators in neutrophil chemotaxis does not reside in its NH2-terminal structure. Mol Pharmacol. 2002;62(1):173-80.

18. Gouwy M, Struyf S, Noppen S, Schutyser E, Springael JY, Parmentier M, et al. Synergy between coproduced CC and CXC chemokines in monocyte chemotaxis through receptor-mediated events. Mol Pharmacol. 2008;74(2):485-95.

19. Cockx M, Gouwy M, Godding V, et al. Neutrophils from patients with primary ciliary dyskinesia display reduced chemotaxis to CXCR2 ligands. Front Immunol. 2017;8:1126.

20. De Buck M, Gouwy M, Berghmans N, et al. COOH-terminal SAA1 peptides fail to induce chemokines but synergize with CXCL8 and CCL3 to recruit leukocytes via FPR2. Blood. 2018;131(4):439-49.

21. William ST, Dunn E, Notarangelo LD, et al. Establishing diagnostic criteria for SCID, leaky SCID, and Omenn syndrome: the primary immune deficiency treatment consortium experience. J Allergy Clin Immunol. 2014;133(4):1092-8.

22. Shiow LR, Paris K, Akana MC, et al. Severe combined immunodeficiency (SCID) and attention deficit hyperactivity disorder (ADHD) associated with a Coronin-1A mutation and a chromosome 16p11.2 deletion. Clin Immunol. 2009;131(1):24-30.

23. Punwani D, Pelz B, Yu J, Arva NC, Schafernak K, Kondratowicz $\mathrm{K}$, et al. Coronin-1A: immune deficiency in humans and mice. $\mathrm{J}$ Clin Immunol. 2015;35(2):100-7.

24. Dadi HK, Simon AJ, Roifman CM. Effect of CD3delta deficiency on maturation of alpha/beta and gamma/delta T-cell lineages in severe combined immunodeficiency. N Engl J Med. 2003;349(19):1821-8.

25. Moshous D, Martin E, Carpentier W, et al. Whole-exome sequencing identifies Coronin-1A deficiency in 3 siblings with immunodeficiency and EBV-associated B-cell lymphoproliferation. J Allergy Clin Immunol. 2013;131(6):1594-603.

26. Meyts I, Bosch B, Bolze A, et al. Exome and genome sequencing for inborn errors of immunity. J Allergy Clin Immunol. 2016;138(4): 957-69.
27. Desmet FO, Hamroun D, Lalande M, et al. Human Splicing Finder: an online bioinformatics tool to predict splicing signals. Nucleic Acid Res. 2009;37(9):e67. https://doi.org/10.1093/nar/gkp215

28. Reese MG, Eeckman FH, Kulp D, Haussler D. Improved splice site detection in genie. J Comp Biol. 1997;4(3):311-23.

29. Nakayama T, Fujisawa R, Izawa D, Hieshima K, Takada K, Yoshie $\mathrm{O}$. Human B cells immortalized with Epstein-Barr virus upregulate CCR6 and CCR10 and downregulate CXCR4 and CXCR5. J Virol. 2002;76(6):3072-7.

30. Dusi S, Nadalini KA, Donini M, et al. Nicotinamide-adenine dinucleotide phosphate oxidase assembly and activation in EBVtransformed B lymphoblastoid cell lines of normal and chronic granulomatous disease patients. J Immunol. 1998;161(9):4968-74.

31. Ammann AJ, Hong R. Disorders of the T-cell system. In: Stiehm ER, editor. Immuno-logic disorders in infants and children. 3rd ed ed. Philadelphia: W.B. Saunders; 1989. p. 257-315.

32. Liu Z, Man SM, Zhu Q, et al. DOCK2 confers immunity and intestinal colonization resistance to Citrobacter rodentium infection. Sci Rep. 2016;6(27814). https://doi.org/10.1038/srep27814

33. Roos D, de Boer M. Molecular diagnosis of chronic granulomatous disease. Clin Exp Immunol. 2014;175(2):139-49.

34. Roberts AW, Kim C, Zhen L, et al. Deficiency of the hematopoietic cell-specific Rho family GTPase Rac2 is characterized by abnormalities in neutrophil function and host defense. Immunity. 1999;10(2):183-96.

35. Williams DA, Tao W, Yang F, et al. Dominant negative mutation of the hematopoietic-specific Rho GTPase, Rac2, is associated with a human phagocyte immunodeficiency. Blood. 2000;96(5):1646-54.

36. Ambruso DR, Knall C, Abell AN, et al. Human neutrophil immunodeficiency syndrome is associated with an inhibitory Rac2 mutation. Proc Natl Acad Sci U S A. 2000;97(9):4654-9.

37. Gu Y, Filippi MD, Cancelas JA, Siefring JE, Williams EP, Jasti AC, et al. Hematopoietic cell regulation by Rac1 and Rac2 guanosine triphosphatases. Science. 2003;302(5644):445-9.

38. Alkhairy OK, Rezaei N, Graham RR, et al. RAC2 loss-of-function mutation in 2 siblings with characteristics of common variable immunodeficiency. J Allergy Clin Immunol. 2015;135(5):1380-4 e1-5.

39. Kuhns D, Fink DL, Choi U, et al. Cytoskeletal abnormalities and neutrophil dysfunction in WDR1 deficiency. Blood. 2016;128(17): 2135-43.

40. Lanzi G, Moratto D, Vairo D, Masneri S, Delmonte O, Paganini T, et al. A novel primary human immunodeficiency due to deficiency in the WASP-interacting protein WIP. J Exp Med. 2012;209(1):29-34.

41. Chae HD, Lee KE, Williams DA, Gu Y. Cross-talk between RhoH and Rac1 in regulation of actin cytoskeleton and chemotaxis of hematopoietic progenitor cells. Blood. 2008;111(5):2597_605.

42. McGhee $\mathrm{S}$, Chatila TA. DOCK8 immune deficiency as a model for primary cytoskeletal dysfunction. Dis Markers. 2010;29(3-4):151-6.

43. Janssen E, Tohme M, Hedayat M, Leick M, Kumari S, Ramesh N, et al. A DOCK8-WIP-WASp complex links T cell receptors to the actin cytoskeleton. J Clin Invest. 2016;126(10):3837-51.

44. Notarangelo L, Ochs HD. Wiskott-Aldrich syndrome: a model for defective actin reorganization, cell trafficking and synapse formation. Curr Opin Immunol. 2003;15(5):585-91.

45. Badolato R, Sozzani S, Malacarne F, et al. Monocytes from Wiskott-Aldrich patients display reduced chemotaxis and lack of cell polarization in response to monocyte chemoattractant protein-1 and formyl-methionyl-leucyl-phenylalanine. J Immunol. 1998;161(2):1026-33.

46. Brigida I, Zoccolillo M, Cicalese MP, et al. T cell defects in patients with ARPC1B germline mutations account for their combined immunodeficiency. Blood. 2018;132(22):2362-74 


\section{Affiliations}

Leen Moens ${ }^{1} \cdot$ Mieke Gouwy ${ }^{2}$ Barbara Bosch ${ }^{3,4}$ - Oleksandr Pastukhov ${ }^{5}$ - Alejandro Nieto-Patlàn ${ }^{6,7,8}$ • Ulrich Siler ${ }^{5}$. Giorgia Bucciol $^{1,3}$. Djalila Mekahli ${ }^{9,10}$. François Vermeulen ${ }^{3}$ - Lars Desmet ${ }^{11}$ - Sophie Maebe ${ }^{11} \cdot$ Helena Flipts $^{12}$. Anniek Corveleyn ${ }^{12}$. Despina Moshous ${ }^{13,14}$. Pierre Philippet ${ }^{15}$. Stuart G. Tangye ${ }^{16,17}$. Bertrand Boisson ${ }^{4,6,7}$. Jean-Laurent Casanova ${ }^{4,6,7,13,18}$ • Benoit Florkin ${ }^{19} \cdot$ Sofie Struyf $^{2}$. Janine Reichenbach ${ }^{5,20,21}$. Jacinta Bustamante ${ }^{4,6,7,22} \cdot$ Luigi D. Notarangelo ${ }^{23} \cdot$ Isabelle Meyts ${ }^{1,3}$ (D)

1 Laboratory for Inborn Errors of Immunity, Department of Immunology and Microbiology, KU Leuven, Leuven, EU, Belgium

2 Laboratory of Molecular Immunology, Department of Microbiology and Immunology, Rega Institute for Medical Research, University of Leuven, Leuven, EU, Belgium

3 Department of Pediatrics, University Hospitals Leuven, Leuven, EU, Belgium

4 St. Giles Laboratory of Human Genetics of Infectious Diseases, Rockefeller Branch, Rockefeller University, New York, USA

5 Institute for Regenerative Medicine associated group, University of Zürich, Zürich, Switzerland

6 Laboratory of Human Genetics of Infectious Diseases, Necker Branch, INSERM U1163, Necker Hospital for Sick Children, Paris, EU, France

7 Paris Descartes University, Imagine Institute, Paris, EU, France

8 Departamento de Inmunología, Escuela Nacional de Ciencias Biológicas, Instituto Politécnico Nacional, ENCB-IPN, México, Mexico

9 Laboratory of Organ Systems, Department of Development and Regeneration, KU Leuven, Leuven, EU, Belgium

10 Department of Pediatric Nephrology, University Hospitals Leuven, Leuven, EU, Belgium

11 Clinical Division and Laboratory of Intensive Care Medicine, Department of Cellular and Molecular Medicine, University Hospitals Leuven, KU Leuven, Leuven, EU, Belgium
12 Center for Human Genetics, University Hospitals Leuven, Leuven, EU, Belgium

13 Pediatric Hematology-Immunology Unit, Necker Hospital for Sick Children, AP-HP, Paris, EU, France

14 INSERM UMR1163, University Paris Descartes Sorbonne Paris Cité, Institut Imagine, Paris, EU, France

15 Division of Pediatric Hematology Oncology, Centre Hospitalier Chrétien, Montegnée, Liege, EU, Belgium

16 Immunology Division, Garvan Institute of Medical Research, Darlinghurst, Sydney, New South Wales 2010, Australia

17 St. Vincent's Clinical School, Faculty of Medicine, University of NSW Sydney, Darlinghurst, New South Wales 2010, Australia

18 Howard Hughes Medical Institute, New York, NY, USA

19 Immuno-Hémato-Rhumatologie Pédiatrique, Service de Pédiatrie, CHR Citadelle, Liège, EU, Belgium

20 Centre for Applied Biotechnology and Molecular Medicine, University of Zürich, Zürich, Switzerland

21 Zurich Centre for Integrative Human Physiology, Zürich, Switzerland

22 Study Centre for Immunodeficiency, Necker Hospital for Sick Children, Paris, EU, France

23 Laboratory of Clinical Immunology and Microbiology, National Institute of Allergy and Infectious Diseases, National Institutes of Health, Bethesda, USA 